\title{
There is No Remarkable Difference Between Pedicle Screw and Hybrid Construct in the Correction of Lenke Type-1 Curves
}

\author{
Ulas SERARSLAN ${ }^{1}$, Emin ALICl ${ }^{2}$, Omer AKCALI ${ }^{3}$, Can KOSAY ${ }^{3}$, Meric UNAL ${ }^{4}$, Alper GULTEKIN¹ \\ ${ }^{1}$ Derince Education and Research Hospital, Department of Orthopaedics and Traumatology, Kocaeli, Turkey \\ ${ }^{2}$ Emeritus Professor of Orthopaedics and Traumatology, Izmir, Turkey \\ ${ }^{3}$ Dokuz Eylul University, Faculty of Medicine, Department of Orthopaedics and Traumatology, Izmir, Turkey \\ ${ }^{4}$ Suleyman Demirel University, Faculty of Medicine, Department of Sports Medicine, Isparta, Turkey
}

This study has been presented as an oral presentation at the $20^{\text {th }}$ Turkish Orthopaedics and Traumatology Congress between 23 and 28 October 2007 , at Ankara, Turkey.

\section{ABSTRACT}

AIM: To compare the deformity correction success of segmental pedicle screw and hybrid instrumentation for the treatment of Lenke type-1 adolescent idiopathic scoliosis (AIS) curves.

MATERIAL and METHODS: Surgically treated Lenke type-1 scoliosis patients were retrospectively evaluated and data of 26 patients treated with hybrid instrumentation were included in the first group. In this group, all patients had been operated with hooks at the thoracic part and transpedicular screws at the lumbar part. The second group included 26 patients operated with all segment transpedicular screws. Cobb angles of curves, flexibility, apical vertebral translation (AVT), coronal body balance, kyphosis and lordosis were measured. All measurements and correction ratios were compared between the groups.

RESULTS: There were no significant differences between the two groups for preoperative thoracic and lumbar Cobb angles, thoracic and lumbar curve flexibility, coronal balance, AVT, kyphosis and lordosis. However, the postoperative thoracic correction ratio was significantly different between the two groups.

CONCLUSION: Segmental screw instrumentation had better results for thoracic curve correction than hybrid instrumentation for the treatment of Lenke type-1 curves. Good results may be achieved with both techniques to provide sagittal balance.

KEYWORDS: Instrumentation, Posterior surgery, Scoliosis surgery

\section{INTRODUCTION}

A dolescent idiopathic scoliosis (AIS) is a complex 3-dimensional disorder characterized by a lateral spinal curve in the coronal plane, thoracic hypokyphosis in the sagittal plane and vertebral rotation in the transverse plane (1). Lenke type-1, primary main thoracic curve pattern, is the most common spinal deformity pattern in AIS (5).

The primary goals in the surgical management of these types of deformities are to achieve a solid arthrodesis, to prevent progression, and to provide three-plane correction (5). Surgical treatment options include thoracoscopic anterior spinal fusion, open anterior spinal fusion and posterior spinal fusion (5). The aim of posterior fusion is to achieve a stable, wellbalanced spine in the coronal and also the sagittal planes (1). Posterior spinal fusion with instrumentation has been shown to achieve reliable deformity correction, solid arthrodesis and low complication rates (5). In the sagittal plane, results of posterior fusion are confusing regarding correction of thoracic hypokyphosis (1). 
Hybrid instrumentation using pedicle hooks and screws is the common technique. However, in the last few decades, all screw instrumentation has become a popular technique for posterior spinal instrumentation. All screw instrumentation theoretically provides stronger biomechanical fixation and improves three-dimensional correction and maintenance of correction (2).

There are few studies to compare segmental all screw and hybrid instrumentation $(1,2,6,9)$. Confusing results have been reported in the literature concerning the comparison of all screw and hybrid instrumentation. The mixed results reflect the heterogenicity of the population and treated curves included in these studies (2).

Our hypothesis is that all screw instrumentation provides better deformity correction in all three planes of Lenke type1 deformities. The aim of this study was to compare the deformity correction success of segmental pedicle screw and hybrid instrumentation for the treatment of Lenke type-1 AIS curves.

\section{MATERIAL and METHODS}

\section{Subjects}

Surgically treated Lenke type-1 scoliosis patients were retrospectively evaluated and data of 26 patients who were treated with hybrid instrumentation were included in the first group. The second group was formed of 26 consecutive patients who were operated with all segment transpedicular screws. All 52 cases were operated between 1991 and 2006. In the hybrid group, pedicle screws were placed to lumbar vertebrae and hooks were placed to thoracic vertebrae (Figure 1 ), and these patients were operated before 2000. In the screw group, segmental pedicle screws were placed to thoracic and lumbar vertebrae (Figure 2). All patients in the screw group were operated after 2000 . Lenke type-1 curves are primary thoracic curves and these patients are suitable for selective thoracic fusion. For this reason, selective thoracic fusion between T4 and T12 or L1 were performed to all patients in both groups. All cases were operated by a single senior orthopaedic spine surgeon at a single stage.

\section{Radiological Evaluation}

Preoperative and early postoperative (two months after operation) weight-bearing posteroanterior (PA), lateral and right and left bending vertebral radiograms were taken for radiological measurements (Figure 3 ). Cobb angles of major and minor curves were measured on weight-bearing PA radiograms preoperatively and postoperatively. Postoperative correction percentages were calculated with the formula below:

Correction Percentage $=($ Preoperative Cobb angle -

Postoperative Cobb angle) / Preoperative Cobb angle x 100.

For the evaluation of flexibility of curves, right and left bending PA vertebral radiograms were used. The flexibility percentage of the curves was calculated with the formula below:

Flexibility Percentage $=(\mathrm{PA}$ Cobb Angle - Bending Cobb Angle) / PA Cobb Angle x 100.

Apical vertebral translation (AVT) was measured from PA radiograms. AVT was the distance between the central sacral longitudinal line and the midpoint of the apical vertebral
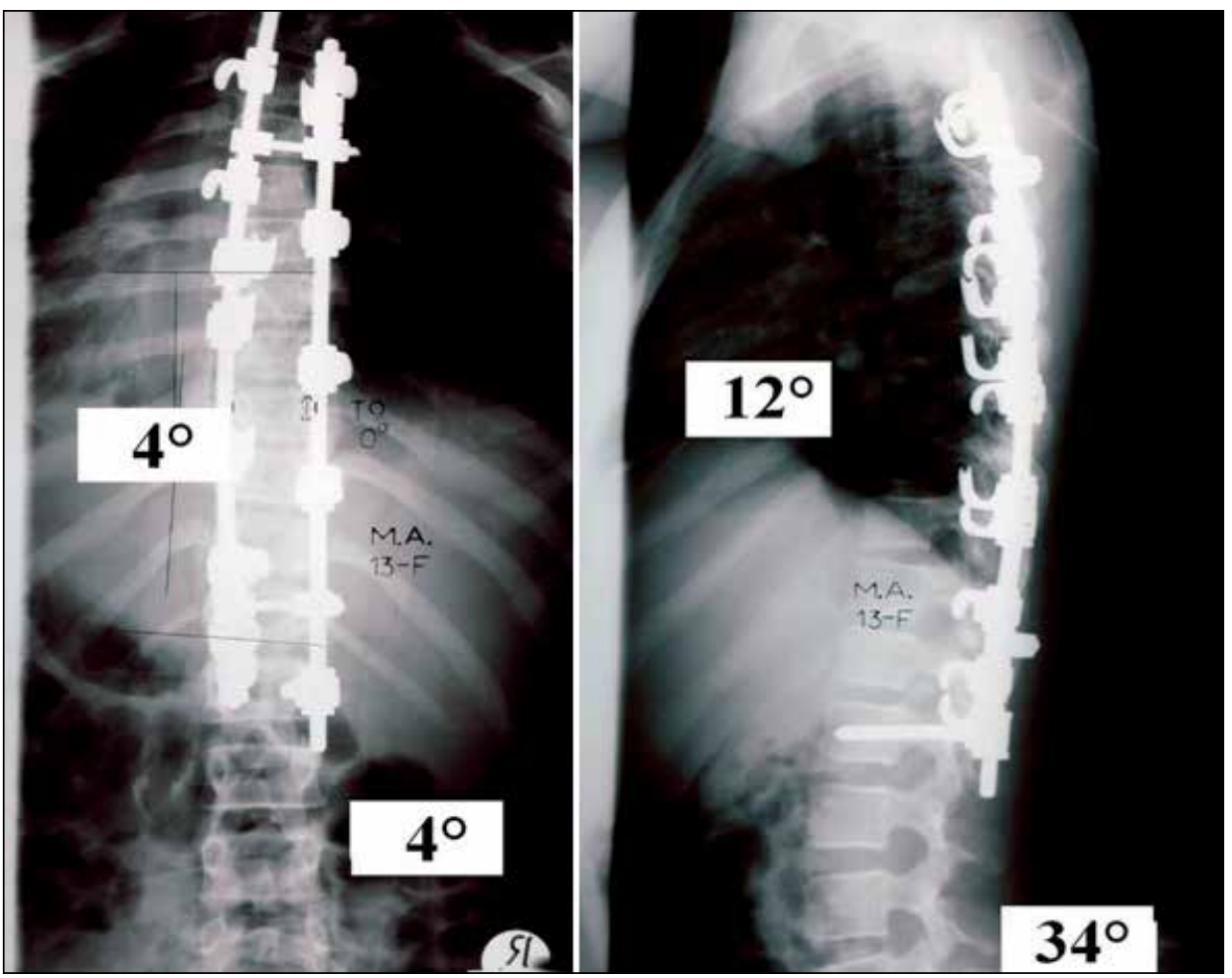

Figure 1: Postoperative AP (left) and lateral (right) radiograms of a patient from hybrid group. 
body. General coronal balance was also measured. It was the distance between the longitudinal line that passes from the middle point of the $7^{\text {th }}$ cervical vertebra and the longitudinal line the passes from the central sacral point.
Thoracic kyphosis was measured from the weight-bearing lateral vertebral radiograms. It was the angle between the upper end plate of the $5^{\text {th }}$ thoracic and the lower end plate of the $12^{\text {th }}$ thoracic vertebra. Lumbar lordosis was also measured. It was the angle between the upper end plate of the $1^{\text {th }}$ lumbar vertebra and the lower end plate of the $5^{\text {th }}$ lumbar vertebra.
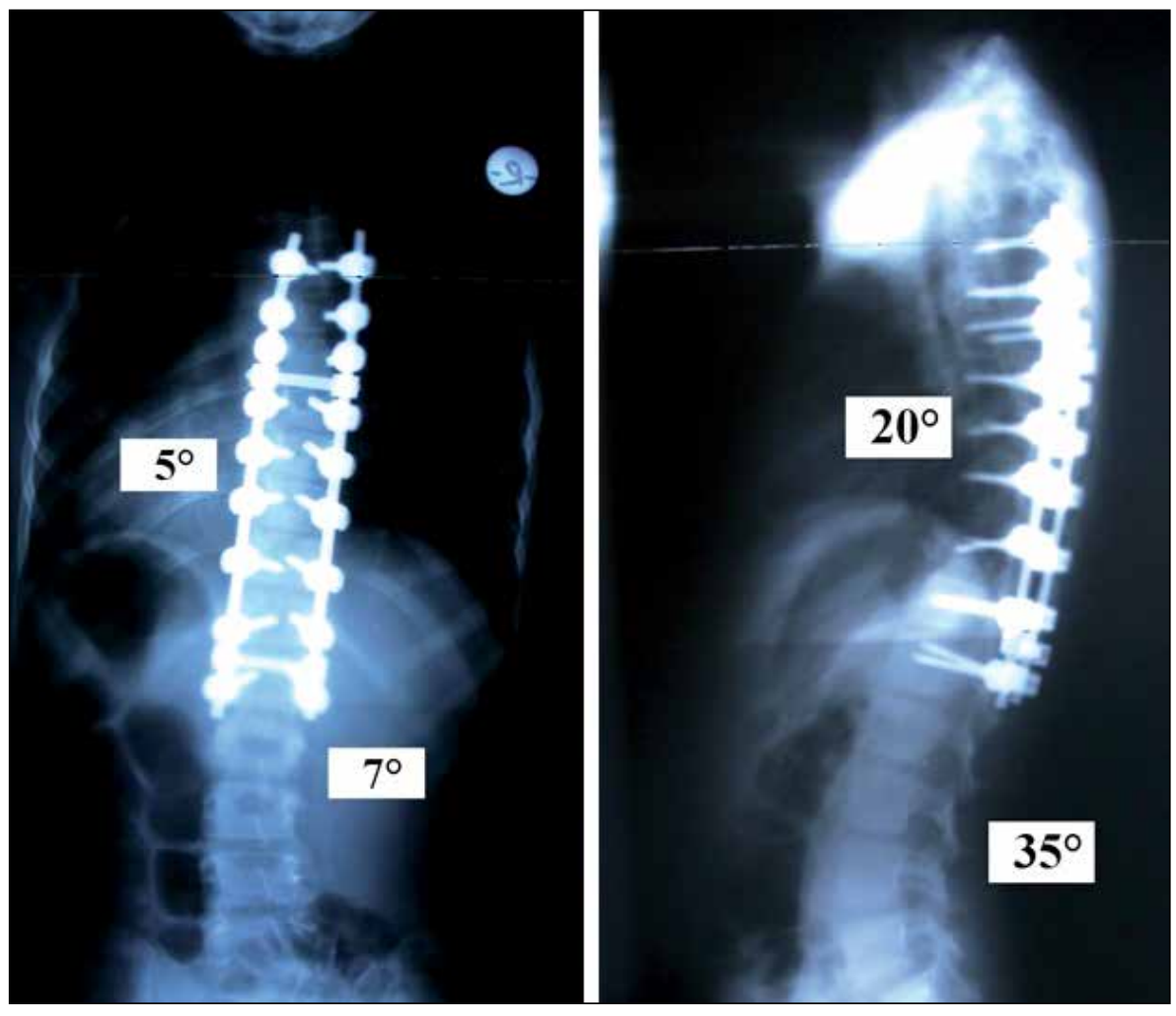

Figure 2: Postoperative AP (left) and lateral (right) radiograms of a patient from screw group.
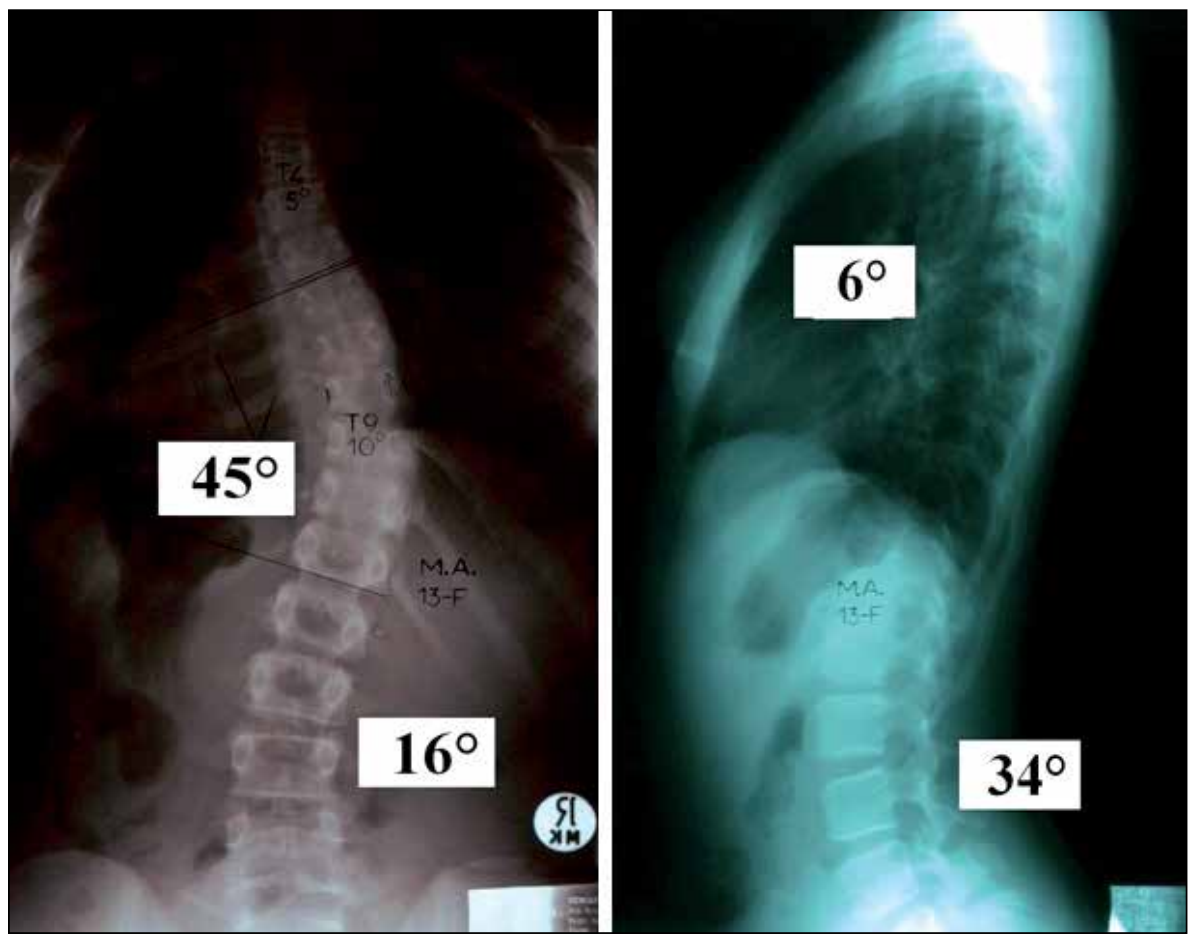

Figure 3: Weight-bearing AP (left) and lateral (right) radiograms of Lenke type-1 AIS. 


\section{Statistical Analysis}

All data were entered to computer using the StatPlus software (version 3.9.8.0, AnalystSoft, Vancouver, Canada). Preoperative and postoperative comparisons inside groups were performed with the paired-samples t test. Preoperative and postoperative comparisons between two groups were performed with the Mann-Whitney $U$ test. $p<0.05$ was accepted as statistically significant.

\section{RESULTS}

The screw group had 20 female and six male patients. The mean age at operation time was 13.8 years (10-20 years). The mean operation time was 102 minutes (79-146) and the mean blood loss was 2.5 units in both groups with no significant differences between the groups. There was no neurologic or major complication in any patient. According to the Lenke classification, the lumbar variable was type-A in 17, type-B in four and type-C in five patients respectively. 19 patients had normal thoracic kyphosis (T5-T12) $\left(10^{\circ}-40^{\circ}\right)$, five patients had hypokyphosis $\left(<10^{\circ}\right)$ and two patients had hyperkyphosis $\left(>40^{\circ}\right)$. The hybrid group had 17 female and nine male patients. The mean age at operation time was 14.4 years (11-18 years). According to the Lenke classification, the lumbar variable was type- $A$ in 18 , type- $B$ in seven and type- $C$ in one patient respectively. Fourteen patients had normal thoracic kyphosis (T5-T12) $\left(10^{\circ}-40^{\circ}\right)$, seven patients had hypokyphosis $\left(<10^{\circ}\right)$ and five patients had hyperkyphosis $\left(>40^{\circ}\right)$ (Table I).

There were no significant differences between the two groups for preoperative thoracic Cobb angle, thoracic curve flexibility, preoperative lumbar Cobb angle, lumbar curve flexibility, coronal plane balance, AVT, preoperative kyphosis angle and lumbar lordosis angle.

In the screw group; there were statistically significant improvements between preoperative and postoperative thoracic Cobb angles $(p<0.0001)$, lumbar Cobb angles $(p<0.0001)$, coronal plane balance $(p=0.01)$, AVT $(p<0.0001)$ and lumbar lordosis angle $(p=0.01)$ (Table II). Five patients that had hypokyphosis and two patients that had hyperkyphosis became normal postoperatively.

In the hybrid group; there were statistically significant improvements between preoperative and postoperative thoracic Cobb angles $(p<0.0001)$, Lumbar Cobb angles $(p<0.0001)$, coronal plane balance (0.02), and AVT (0.0001). There was no statistically significant difference for lumbar lordosis angle $(p=0.45)$ (Table III). Four of the seven patients that had hypokyphosis and four of the five patients that had hyperkyphosis became normal postoperatively. One patient that had normal thoracic kyphosis became hypokyphotic postoperatively.

In the comparison of the screw and hybrid groups, there was a significant difference between the groups for postoperative thoracic correction percentage $(p=0.016)$. There were no statistically significant differences between the two groups for postoperative lumbar correction percentage $(p=0.86)$, coronal plane balance $(p=0.86)$, AVT $(p=0.52)$, thoracic kyphosis change $(p=0.84)$ and lumbar lordosis change $(p=0.25)$ (Table IV).

\section{DISCUSSION}

This study focused on the effect of two instrumentation types on coronal and sagittal plane deformity correction of Lenke type-1 AIS curves. Comparisons were matched with regard basically to radiographic parameters. All patients included to the study had Lenke type-1 curves, so group heterogenicity regarding curve pattern was minimized. All surgeries were performed by one senior orthopaedic spine surgeon and technical differences were also minimized. Both of the groups had very similar demographic features and preoperative curve patterns. Most of previous studies had failed to address some variables like group heterogenicity, technical differences and distinct curve patterns (2).

Pedicle screw fixation is considered biomechanically better than hook fixation because it uses the pedicle as an anchor (2). It may also allow improved deformity correction and preserves more motion because of reducing fused segments (7). The results of this study confirm the corrective ability of segmental screw instrumentation on the coronal and sagittal planes. Segmental screw instrumentation had better results on thoracic curve correction than hybrid instrumentation for the treatment of Lenke type-1 curves.

Crawford et al., in their recent study in 2013, compared segmental and hybrid instrumentations. They concluded that segmental pedicle screw instrumentation provided better results for the maintenance of correction (2).

Kim et al. reported a retrospective comparative study and found better correction ability in segmental screw instrumentation than hybrid instrumentation. Their groups were matched according to age, Lenke type, fusion levels and operative technique but not preoperative curve flexibility (3). Preoperative curve flexibility was considered as an important factor and in our study; groups were also matched according to flexibility. Yilmaz et al. compared hook, hybrid and segmental screw instrumentations. They found better results with segmental screw and hybrid instrumentations than hook instrumentation in major curve coronal correction (9). Rafi et al. reported better results with segmental screw instrumentation for correction of AIS than hybrid instrumentation in their prospective study. They matched groups for operation time and bleeding but not according to the Lenke classification, Cobb angle and curve flexibility (6). Cao et al. evaluated segmental screw instrumentation and hybrid instrumentation according to thoracic kyphosis in their meta-analysis. They concluded in their study that there was a tendency for both instrumentation techniques to restore thoracic kyphosis (1).

Superiority of segmental screw instrumentation was also evaluated in another meta-analysis. Harrington rods, CotrelDubousset (CD) instrumentation and segmental screw instrumentation were included in this meta-analysis. The degree of correction was found better after CD instrumentation compared with segmental screw instrumentation for both thoracic and lumbar curves (4).

Westrick et al. reported that coronal plane correction was not the most important measure because residual coronal plane deformity could be well tolerated. On the contrary, sagittal 
Table I: Preoperative Demographic and Radiological Features of the Groups

\begin{tabular}{llcr}
\hline & & Hybrid Group & Screw Group \\
\hline Gender & Male & 9 & 6 \\
\hline Mean age (years) & Female & 17 & 20 \\
\hline Lumbar variable & & 14.4 years & 13.8 years \\
\hline & Type A & 18 & 17 \\
\hline Thoracic kyphosis & Type B & 7 & 4 \\
\hline & Type C & 1 & 5 \\
\hline & Normal & 14 & 19 \\
\hline
\end{tabular}

Table II: Preoperative and Postoperative Measurements of the Screw Group

\begin{tabular}{lccc}
\hline & Preoperative (mean \pm SD) & Postoperative (mean \pm SD) & p value \\
\hline Thoracic Cobb angle $\left(^{\circ}\right)$ & $57.3 \pm 12.4$ & $9.2 \pm 6.1$ & $<\mathbf{0 . 0 0 0 1}$ \\
\hline Lumbar Cobb angle $\left(^{\circ}\right)$ & $28.7 \pm 9.4$ & $4.6 \pm 4$ & $<\mathbf{0 . 0 0 0 1}$ \\
\hline Thoracic kyphosis angle $\left(^{\circ}\right)$ & $22.8 \pm 12.8$ & $19.9 \pm 5.6$ & $\mathbf{0 . 1 9}$ \\
\hline Lumbar lordosis angle $\left(^{\circ}\right)$ & $35.5 \pm 6.7$ & $30.6 \pm 9.1$ & $\mathbf{0 . 0 1 4}$ \\
\hline Coronal balance $(\mathrm{mm})$ & $17.9 \pm 8.9$ & $11.6 \pm 9.5$ & $\mathbf{0 . 0 1 1}$ \\
\hline AVT $(\mathrm{mm})$ & $50.6 \pm 17.5$ & $11.2 \pm 7.4$ & $<\mathbf{0 . 0 0 0 1}$ \\
\hline
\end{tabular}

Table III: Preoperative and Postoperative Measurements of the Hybrid Group

\begin{tabular}{lccc}
\hline & Preoperative (mean \pm SD) & Postoperative (mean \pm SD) & p value \\
\hline Thoracic Cobb angle $\left(^{\circ}\right)$ & $51 \pm 10.1$ & $13.4 \pm 8.6$ & $<0.0001$ \\
\hline Lumbar Cobb angle $\left(^{\circ}\right)$ & $29 \pm 7.5$ & $6.1 \pm 6.8$ & $<0.0001$ \\
\hline Thoracic kyphosis angle $\left(^{\circ}\right)$ & $25.1 \pm 15.8$ & $21.2 \pm 9.8$ & $\mathbf{0 . 1 9}$ \\
\hline Lumbar lordosis angle $\left(^{\circ}\right)$ & $35.1 \pm 11.5$ & $33.4 \pm 10.6$ & $\mathbf{0 . 0 1 4}$ \\
\hline Coronal balance $(\mathrm{mm})$ & $19.5 \pm 13.4$ & $12.7 \pm 8.6$ & $\mathbf{0 . 0 1 1}$ \\
\hline AVT $(\mathrm{mm})$ & $52 \pm 23.4$ & $15.8 \pm 12.2$ & $<0.0001$ \\
\hline
\end{tabular}

AVT: Apical vertebral translation.

Table IV: Postoperative Comparison of the Screw and Hybrid Groups

\begin{tabular}{lccc}
\hline & Screw Group & Hybrid Group & p value \\
\hline Thoracic correction $(\%)$ & $83 \pm 11$ & $73 \pm 16$ & $\mathbf{0 . 0 1 6}$ \\
\hline Lumbar correction $(\%)$ & $81 \pm 21$ & $79 \pm 21$ & $\mathbf{0 . 8 6}$ \\
\hline Thoracic kyphosis change $\left(^{\circ}\right)$ & $-2.9 \pm 11.1$ & $-3.9 \pm 11.6$ & $\mathbf{0 . 8 4}$ \\
\hline Lumbar lordosis change $\left(^{\circ}\right)$ & $-4.9 \pm 9.4$ & $-1.7 \pm 11.7$ & $\mathbf{0 . 2 5}$ \\
\hline Coronal balance change $(\mathrm{mm})$ & $-6.3 \pm 11.7$ & $-6.8 \pm 14.1$ & $\mathbf{0 . 8 6}$ \\
\hline AVT change $(\mathrm{mm})$ & $39.4 \pm 18.2$ & $-36.2 \pm 19.2$ & $\mathbf{0 . 5 2}$ \\
\hline
\end{tabular}

AVT: Apical vertebral translation. 
alignment and balance has been strongly associated with outcomes of AIS (8). In our study, there was no difference between segmental pedicle instrumentation and hybrid instrumentation when considering sagittal balance. Both techniques gave good results to provide sagittal balance.

In this study, all curves were Lenke type-1 curves. All patients were operated by the same senior orthopaedic spine surgeon. Groups are also standardized according to thoracic Cobb angle, lumbar Cobb angle, thoracic curve flexibility, lumbar curve flexibility, coronal plane balance, AVT, preoperative kyphosis angle and lumbar lordosis angle. The comparisons between groups were therefore highly standardized, in contrast to the literature.

There are some limitations for this study. The numbers of subjects in the groups are not small, but larger clinical series may give a better power ratio. However, hybrid instrumentation is not a widely used technique since thoracic screw applications. Another problem was about the study design. This is a cross-sectional study, and that is why correctional loss in long-term follow-up cannot be evaluated.

\section{CONCLUSION}

There are few studies that compared segmental screw and hybrid instrumentations for treatment of AIS. This study was strictly standardized according to Lenke type (only type-1 curves included), thoracic Cobb angle, lumbar Cobb angle, thoracic curve flexibility, lumbar curve flexibility, coronal plane balance, AVT, preoperative kyphosis angle and lumbar lordosis angle. The results of this study confirm the corrective ability of segmental screw instrumentation on coronal and sagittal planes. Segmental screw instrumentation had better results for thoracic curve correction than hybrid instrumentation for the treatment of Lenke type-1 curves. No difference was found between segmental pedicle instrumentation and hybrid instrumentation when considering sagittal balance. Both techniques gave good results to provide sagittal balance. We showed that the average success with a hybrid system (pedicle screw plus hooks) for the correction of deformity was the same as a pedicle screw system, in contrast to the literature, but we do not know the late results.

\section{REFERENCES}

1. Cao Y, Xiong W, Li F: Pedicle screw versus hybrid construct instrumentation in adolescent idiopathic scoliosis. Spine 39: 800-810, 2014

2. Crawford AH, Lykissas MG, Gao X, Eismann E, Anadio J: Allpedicle screw versus hybrid instrumentation in adolescent idiopathic scoliosis surgery. Spine 38:1199-1208, 2013

3. Kim YJ, Lenke LG, Kim J Bridwell KH, Cho SK, Cheh G, Sides B: Comparative analysis of pedicle screw versus hybrid instrumentation in posterior spinal fusion of adolescent idiopathic scoliosis. Spine 31: 291-298, 2006

4. Lykissas MG, Jain VV, Nathan ST, Pawar V, Eismann EA, Sturm $\mathrm{PF}$, Crawford $\mathrm{AH}$ : Mid- to long-term outcomes in adolescent idiopathic scoliosis following instrumented posterior spinal fusion: A meta-analysis. Spine 38:113-119, 2013

5. Newton PO, Marks MC, Bastrom TP, Betz R, Clements D, Lonner B Crawford A, Shufflebarger H, O'Brien M, Yaszay B; Harms Study Group: Surgical treatment of Lenke 1 main thoracic idiopathic scoliosis. Spine 38: 328-338,2013

6. Rafi S, Munshi N, Abbas A, Shaikh RH, Hashmi I: Comparative analysis of pedicle versus hybrid instrumentation in adolescent idiopathic scoliosis surgery. J Neurosci Rural Pract 7:550-553, 2016

7. Suk SI, Lee CK, Kim WJ, Chung YJ, Park YB: Segmental pedicle screw fixation in the treatment of thoracic idiopathic scoliosis. Spine 20:1399-1405, 1995

8. Westrick ER, Ward WT: Adolescent idiopathic scoliosis: 5-year to 20-year evidence-based surgical results. J Pediatr Orthop 31:61-68, 2011

9. Yilmaz G, Borkhuu B, Dhawale AA, Oto M, Littleton AG, Mason DE, Gabos PG, Shah SA: Comparative analysis of hook, hybrid and pedicle screw instrumentation in the posterior treatment of adolescent idiopathic scoliosis. J Pediatr Orthop 32:490499, 2012 